Santana Castañeda, G., Aguilar Martínez, A.G. (2020). Sitios candidatos para nuevos servicios médicos utilizando técnicas de evaluación multicriterio, en la Zona Metropolitana de Toluca, México. GeoFocus, Revista Internacional de Ciencia y Tecnología de la Información Geográfica, 26, 139-162. http://dx.doi.org/10.21138/GF.602

\title{
SITIOS CANDIDATOS PARA NUEVOS SERVICIOS MÉDICOS UTILIZANDO TÉCNICAS DE EVALUACIÓN MULTICRITERIO, EN LA ZONA METROPOLITANA DE TOLUCA, MÉXICO
}

\author{
${ }^{1 \mathrm{a}}$ GIOVANNA SANTANA CASTAÑEDA \\ MARTÍNEZ iD \\ ${ }^{1}$ Facultad de Geografía. Universidad Autónoma del Estado de México, UAEMEX \\ Ciudad Universitaria, C. U. 50100 Toluca de Lerdo, México \\ ${ }^{2}$ Instituto de Geografía. Universidad Nacional Autónoma de México, UNAM \\ Ciudad Universitaria, C.U., Coyoacán, 04510 Ciudad de México, México. \\ agsantanac@uaemex.mx, badrian@servidor.unam.mx
}

\section{RESUMEN}

La creación de nuevas infraestructuras de servicios médicos supone una alteración en la condición de vida de las personas, ofreciendo nuevas oportunidades para ampliar la atención en la salud de la población y de esta manera mejorar la calidad de salud de la población en la Zona Metropolitana de Toluca.

El objetivo de esta investigación es proporcionar la localización de sitios candidatos que exterioricen estas oportunidades, mediante la sistematización de variables que expresen la aptitud del territorio para obtener las áreas potencialmente más aptas para las nuevas ubicaciones. El método utilizado para evaluar los criterios fue la suma lineal ponderada borrosa, y para determinar las ponderaciones se utilizó las Jerarquías analíticas de Saaty y la técnica de Jerarquía de Malczewski.

Palabras clave: servicios médicos; capacidad de acogida; evaluación multicriterio; Toluca; México.

\section{CANDIDATE LOCATIONS FOR NEW MEDICAL SERVICES BASED ON MULTICRITERIA DECISION ANALYSIS, IN THE TOLUCA METROPOLITAN ZONE}

The creation of new medical service infrastructures means an alteration in people's living conditions, offering new opportunities to extend health care to the population and improve the quality of health in the Toluca Metropolitan Zone. 
Santana Castañeda, G., Aguilar Martínez, A.G. (2020). Sitios candidatos para nuevos servicios médicos utilizando técnicas de evaluación multicriterio, en la Zona Metropolitana de Toluca, México. GeoFocus, Revista Internacional de Ciencia y Tecnología de la Información Geográfica, 26, 139-162. http://dx.doi.org/10.21138/GF.602

The objective of this study is to determine the location of candidate areas that show these opportunities, systematizing the variables that express the territory's aptitude for obtaining the areas that are potentially most appropriate for the new locations. The method used to evaluate the criteria was the fuzzy weighted linear combination. Saaty's Analytical Hierarchies Process and Malczewski's Hierarchy structure were used to determine the weights.

Keywords: healthcare services; territorial aptitude; multicriteria decision analysis; Toluca; México.

\section{Introducción}

A nivel internacional, el análisis de la localización de los servicios públicos ha inspirado el debate sobre la localización, distribución y áreas de influencia, aunado a ello y para fortalecer el estudio de los servicios de salud se suma la teoría de la Geografía de la salud, que aborda la discusión sobre la distribución espacial de las enfermedades, las características físicas y sociales que condicionan la salud humana, así como el análisis sobre los servicios médicos públicos y privados.

Sin embargo, en México a nivel operativo las decisiones que corresponden a la localización de servicios colectivos públicos en particular los que se refieren a la salud humana, se han ajustado a criterios políticos y económicos que regularmente son diferentes a las necesidades de la población y por lo mismo no atiende a toda la población de manera igual o justa.

Es por ello, que la propuesta en el presente artículo para la nueva localización de dichos servicios considera una solución integral, tomando en cuenta los principios de justicia espacial atenta a las necesidades socioespaciales y concretamente la justicia distributiva de los servicios en línea con la normatividad vigente para su correcto equipamiento.

Para determinar las áreas de mayor aptitud locacional, se parte de una consulta a expertos en dónde se concluyó que eran necesarios tres conjuntos de factores: el primero concerniente a la accesibilidad de los centros de salud existentes, el segundo considera la accesibilidad y características físicas del territorio y el tercero relacionado con la demanda y necesidad potencial de las personas. Se analizan los servicios públicos bajo la premisa de que los grupos de estatus alto satisfacen todas o parte de sus necesidades en el sector privado, reduciendo la presión sobre la oferta pública, en tanto que los grupos más desfavorecidos prácticamente proyectan toda su demanda sobre el sector público.

Además, se consideran tres restricciones: el nuevo servicio no puede localizarse sobre cuerpos de agua, bosques, ni el área de la cobertura actual de los servicios médicos.

Se aborda una metodología basada en la utilización de sistemas de información geográfica (SIG) con técnicas de evaluación multicriterio (EMC), como herramienta de apoyo para la obtención de sitios con mayor aptitud para los nuevos servicios médicos; a continuación, se abordan los métodos de evaluación multicriterio.

Los análisis EMC ofrecen la oportunidad de obtener un análisis equilibrado de todas las facetas de los problemas de planificación particularmente debido a que varios efectos intangibles 
Santana Castañeda, G., Aguilar Martínez, A.G. (2020). Sitios candidatos para nuevos servicios médicos utilizando técnicas de evaluación multicriterio, en la Zona Metropolitana de Toluca, México. GeoFocus, Revista Internacional de Ciencia y Tecnología de la Información Geográfica, 26, 139-162. http://dx.doi.org/10.21138/GF.602

como los sociales y las repercusiones ambientales pueden ser considerados cabalmente (Nijkamp \& Van Delft, 1977:5).

Diferentes autores han abordado los modelos de evaluación multicriterio como Diamond \& Wright (1988:206); Janssen \& Rietveld (1990:129); Church (1999:293); Eastman, Jiang, \& Toledano (1998:227); Malczewski (1999:224); Gómez y Barredo (2005:123); entre otros. Todos ellos coinciden en que estos modelos proporcionan un amplio conjunto de técnicas y procedimientos para la resolución de problemas de decisión, estructuración, diseño, evaluación y priorización de decisiones alternativas. En el nivel más simple, la combinación de los SIG con las técnicas de EMC puede ser pensado como un proceso que transforma y combina los datos geográficos y valora juicios también llamados preferencias del decisor para generar conocimiento y toma de decisiones.

En este sentido, la toma de decisiones multicriterio debe ser entendida como un "mundo de conceptos, aproximaciones, modelos y métodos, para auxiliar a los decisores a analizar, evaluar, ordenar, jerarquizar, seleccionar o rechazar objetos, en base a una evaluación (expresada por puntuaciones, valores o intensidades de preferencia) de acuerdo con varios criterios" como lo menciona Colson \& De Bruin en 1989:1201; y según corresponda con el objetivo y la necesidad de resolver el problema se utilizará alguno.

En términos generales, el problema en la EMC es seleccionar la alternativa que "mejor" satisfaga las preferencias del decisor, por lo tanto, este problema descarta la posibilidad de alcanzar una solución óptima debido a que el concepto de óptimo carece de sentido en todo el ámbito de la decisión multicriterio, así que debemos estar claros en que solo vamos a encontrar ese "mejor" entre comillas debido a que su definición está abierta a diversas interpretaciones más o menos racionales y depende en gran medida de los decisores, los cuales siempre llegan a una solución compromiso (Gómez \& Barredo, 2005:45).

\section{a) Métodos de evaluación multicriterio}

Existen diferentes métodos de evaluación multicriterio divididos de la siguiente manera:

- Técnicas no compensatorias:

- Dominancia

- Método conjuntivo

- Método disyuntivo

- Método lexicográfico

- Técnicas compensatorias:

- Aditivas

- Suma lineal ponderada

- Análisis de concordancia

- Procedimiento de jerarquías analíticas (AHP Analytical Hierarchy

Process)

- Sistema de propiedades multiatributo

- De punto ideal

- Basadas en la aproximación al punto ideal 
Santana Castañeda, G., Aguilar Martínez, A.G. (2020). Sitios candidatos para nuevos servicios médicos utilizando técnicas de evaluación multicriterio, en la Zona Metropolitana de Toluca, México. GeoFocus, Revista Internacional de Ciencia y Tecnología de la Información Geográfica, 26, 139-162. http://dx.doi.org/10.21138/GF.602

- Técnicas borrosas

- TOPSIS (Technique for Order Preference by Similarity to Ideal Solution)

- Suma lineal ponderada

- Promedio ponderado ordenado (Ordered Weighted Averaging)

Fuente: Elaboración propia, en base a Barba \& Pomerol (1995:105); Jankowski (1995:252); Malczewski (1999:150) y Gómez \& Barredo (2005:84).

Las técnicas no compensatorias son todas aquellas que no permiten la compensación proporcional entre los criterios, por ejemplo, la técnica de dominancia elimina sitios candidatos a partir de la puntuación que se le asigna a los criterios, el método conjuntivo también llamado disyuntivo realiza esa eliminación a partir de umbrales establecidos para los criterios, el método lexicográfico realiza la eliminación a partir de la ordenación de los criterios y la comparación de las alternativas.

Por el contrario, en las técnicas compensatorias, si existe un valor no adecuado en un criterio este puede ser compensado con un valor adecuado de otro criterio (principio de los vasos comunicantes ${ }^{1}$ ).

Por ejemplo, el método de suma lineal ponderada asume que los valores se pueden descomponer y semejar un modelo aditivo por lo que los juicios que evalúan cada alternativa según cada criterio admiten representaciones numéricas sobre una escala de valores reales, por lo que, el promedio ponderado de cada alternativa refleja el valor que cada una tiene para el decisor, por lo tanto con su ordenación en base a los valores es posible determinar la mejor alternativa (que sería la suma ponderada), esta importancia relativa de cada criterio es caracterizada por un parámetro llamado "peso" (Pomerol \& Barba, 1993:134).

El método de las jerarquías analíticas desarrollado por (Saaty, 1990:10) consiste esencialmente en precisar la compresión lógica de problemas complejos que utilizan una estructura jerárquica, de manera general tiene tres conceptos fundamentales: el primero consiste en la estructuración del problema (objetivo, criterios y alternativas), el segundo incluye las comparaciones por pares de elementos del mismo nivel de la jerarquía con respecto a cada criterio del nivel superior y en el tercero de manera vertical se sintetizan los juicios sobre los diferentes niveles de la jerarquía.

Por otro lado, el método TOPSIS parte del concepto del ideal en dónde su precursor (Zeleny, 1982:153) coloca este concepto como la parte más importante en la propuesta de solución compromiso y por lo tanto, sería la alternativa más próxima al ideal. Este método propuesto por (Hwang \& Yoon, 1981:139) trabaja con el ideal y/o con el anti-ideal o con la mezcla de los dos, cuyo objetivo es la evaluación de las alternativas; en este caso sería una alternativa teórica que reuniera dos condiciones: mínimo impacto y máxima aptitud.

\footnotetext{
${ }^{1}$ Vasos comunicantes, es el nombre que recibe un conjunto de recipientes comunicados por su parte inferior y que contienen un líquido homogéneo, se observa que cuando el líquido está en reposo alcanza el mismo nivel en todos los recipientes, sin influir la forma y volumen de estos.
} 
Santana Castañeda, G., Aguilar Martínez, A.G. (2020). Sitios candidatos para nuevos servicios médicos utilizando técnicas de evaluación multicriterio, en la Zona Metropolitana de Toluca, México. GeoFocus, Revista Internacional de Ciencia y Tecnología de la Información Geográfica, 26, 139-162. http://dx.doi.org/10.21138/GF.602

Las técnicas borrosas (fuzzy o difusas) evalúan los valores o posibilidades de pertenencia al grupo difuso o borroso de las celdas de datos basándose en cualquiera de las siguientes tres funciones de pertenencia: sigmoideo ( $S$-shaped $)$, en forma de $\mathrm{J}(J$-shaped $)$, lineal y/o a través de una pertenencia definida por el usuario. Aunado a ello se utilizan las variantes de aumento o disminución monótonas, ya sean simétricas o asimétricas: Tseng \& Cerry (1992:45); Abdullah (2013:132) y Abdullah \& Jamal (2011:350).

La función de pertenencia borrosa sigmoidea tiene la caracteristica de estar definida por sus límites inferior a, superior $\mathbf{b}$ y el valor $\mathbf{m}$ que es el punto de inflexión, siendo $\mathbf{a}<\mathbf{m}<\mathbf{b}$. El crecimiento es más lento cuanto mayor sea la distancia entre a y b. Por ejemplo, cuando $m=(a+b) / 2$ se obtiene la gráfica de la Figura 1 (siendo $\mathrm{a}=2 ; \mathrm{b}=10 ; \mathrm{m}=(2+10) / 2=6)$.
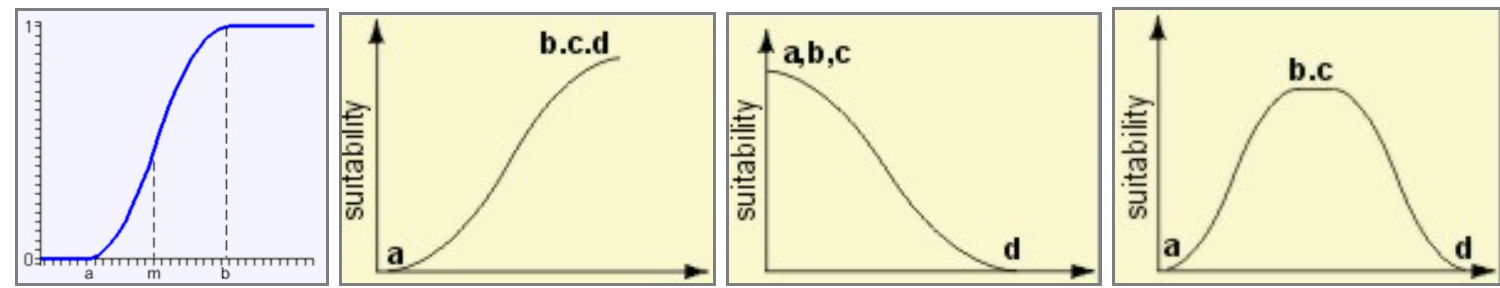

Figura 1. Función de pertenencia borrosa sigmoidea

Fte. Elaboración propia, en base a Idrisi, 2015

La función de pertenencia borrosa lineal o trapezoidal es definida por sus límites inferior a, superior $\mathbf{d}$ y los límites de soporte inferior $\mathbf{b}$ y superior $\mathbf{c}$, tal que $\mathbf{a}<\mathrm{b}<\mathrm{c}<\mathrm{d}$. En este caso, si los valores de b y c son iguales se obtiene una función triangular; como se muestra en la Figura 2
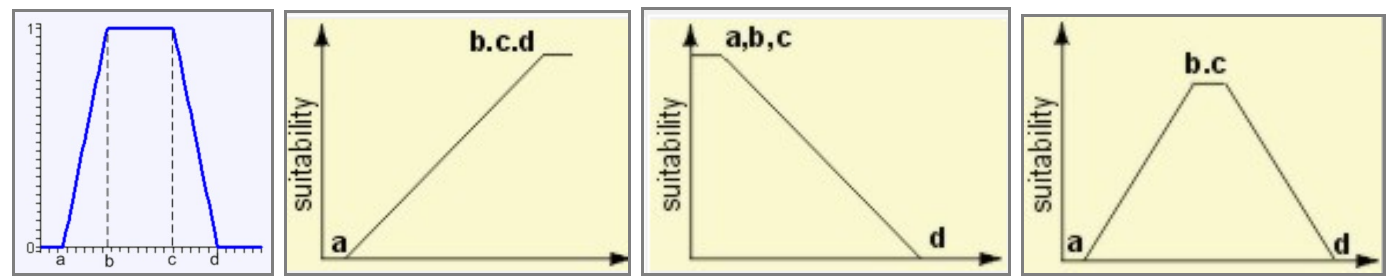

Figura 2. Función de pertenencia borrosa lineal

Fte. Elaboración propia, en base a Idrisi, 2015

La función de pertenencia borrosa de tipo J-Shaped se caracteriza por un rapido crecimiento a partir de a hacía b, como se muestra en la Figura 3. 


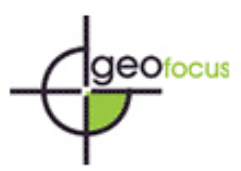

Santana Castañeda, G., Aguilar Martínez, A.G. (2020). Sitios candidatos para nuevos servicios médicos utilizando técnicas de evaluación multicriterio, en la Zona Metropolitana de Toluca, México. GeoFocus, Revista Internacional de Ciencia y Tecnología de la Información Geográfica, 26, 139-162. http://dx.doi.org/10.21138/GF.602
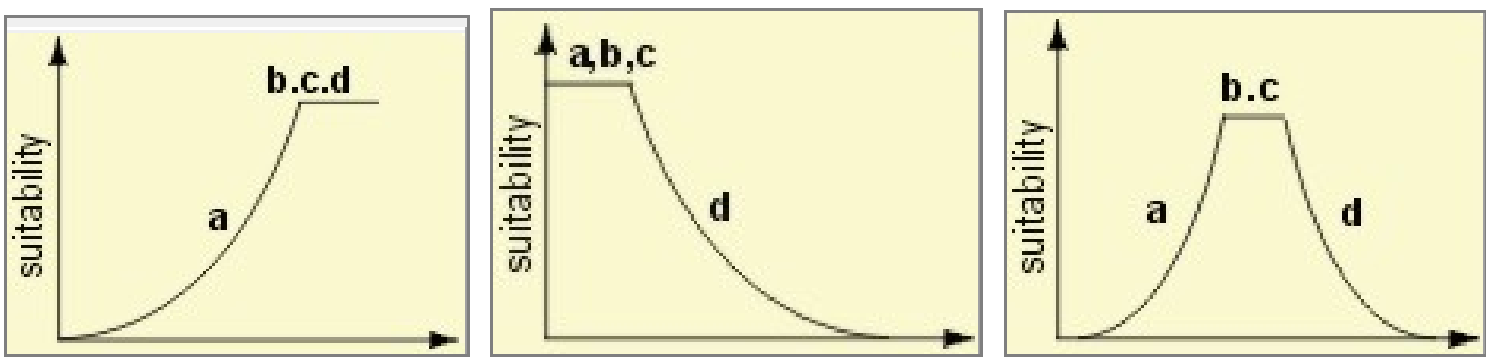

Figura 3. Función de pertenencia borrosa J-Shaped

Fte. Elaboración propia, en base a Idrisi, 2015

A estas funciones se les asigna valores mínimos y máximos como límites de la normalización, lo que significa que a todo lo que esté por debajo del valor mínimo se le asignará un 0 y a todo lo que esté por encima del máximo se le asignará un valor de 1, 100 o 255, según se normalice.

La técnica de evaluación multicriterio se aplica a aquellos problemas en los que se busque la mejor alternativa, que pueden ser dominantes cuando sus evaluaciones son mejores o al menos iguales, u otras alternativas que pueden ser nominales cuando las primeras no son satisfactorias.

En el método de promedio ponderado ordenado (Ordered Weighted Averaging) los pesos no se otorgan a un criterio dado, sino que son jerarquizados (se numeran $1,2,3$, etc.) y para cada alternativa se destina el peso w1 a la mayor utilidad, el peso w2 al segundo, y así subsecuentemente, por lo que cada alternativa tiene su propio juego de peso, y si el valor de sus criterios cambia, su juego de pesos también puede cambiar.

Considerando lo antes mencionado, las técnicas no compensatorias demandan una jerarquización ordinal de los criterios basada en las prioridades de la evaluación, o bien, en el orden de importancia de los criterios sin establecer un peso de manera cuantitativa; mientras que las técnicas compensatorias requieren que se especifiquen los pesos de los criterios como valores cardinales o funciones de prioridad de manera numérica en escala de razón (Galacho \& Ocaña, 2006:1510).

Debido a ello no se observa ningún inconveniente en utilizarlas de manera combinada: las compensatorias como factores (borrosos y normalizados de 0 a 255) y las no compensatorias como restricciones, debido a que los criterios que se restringen implican dentro de los juicios que orientan esta investigación una limitación absoluta y no puede compensarse con otro factor positivo.

\section{b) Componentes de la evaluación multicriterio}

El componente principal en la evaluación multicriterio es el objetivo, que puede ser uno o varios, los cuales pueden ser complementarios o conflictivos, según el número y tipo de objetivos cambia el procedimiento de combinación de los criterios para encontrar la solución.

El segundo componente son las alternativas, que son cada una de las soluciones posibles a un problema dotadas de ventajas e inconvenientes y que se caracterizan por ser completamente disjuntas y exhaustivas, lo que significa que no cabe concebir alternativas a la mitad de las 
Santana Castañeda, G., Aguilar Martínez, A.G. (2020). Sitios candidatos para nuevos servicios médicos utilizando técnicas de evaluación multicriterio, en la Zona Metropolitana de Toluca, México. GeoFocus, Revista Internacional de Ciencia y Tecnología de la Información Geográfica, 26, 139-162. http://dx.doi.org/10.21138/GF.602

enumeradas; si dichas alternativas quisieran considerarse deben añadirse como carácter individual a la lista de alternativas. Hay que entender que las alternativas son la base de la decisión y, por lo tanto, se debe tener particular atención en su estructuración. Considerando lo anterior, las alternativas deben tener las siguientes características:

a) Deben ser exhaustivas, para que no falte ningún criterio que permita discriminar las alternativas.

b) Deben ser coherentes, las preferencias globales del decisor son coherentes con las preferencias según cada criterio. Por ejemplo, si dos alternativas ( $a$ y $b)$ tienen la misma calificación en todos los criterios, implica una preferencia global de $a$ respecto a $b$.

c) No deben ser redundantes. La redundancia existe si la supresión de uno solo de los criterios no implica que el subconjunto de los restantes viole alguna de las anteriores propiedades, propiedad deseable, pero no esencial, puesto que es un peligro para el resultado duplicar algún hecho.

Los criterios son los distintos aspectos de la realidad que inciden de alguna manera en las ventajas o inconvenientes de las alternativas disponibles como soluciones al problema. Los criterios se dividen en factores $\mathrm{y} / \mathrm{o}$ restricciones. Los tipos de criterios son nombrados factores y restricciones, los primeros son aquellos que aumentan o disminuyen la valoración de la aptitud de una alternativa como solución al problema que pueden ser cuantitativos o cualitativos, mientras que las restricciones son los aspectos que determinan que alternativas son válidas (aceptables) y cuales no como solución al problema; las restricciones son siempre binarias o booleanas, lo que significa que están dadas por valores de 0 o 1 , el 0 donde no es viable y el 1 donde sí lo es (Gómez \& Barredo, 2005:79).

El último componente es la decisión, es decir, la elección de una de las alternativas posibles para solucionar un problema mediante la combinación y ponderación de estos criterios.

\section{c) Métodos de Ponderación}

Para la modelización de los criterios, los factores deben ser normalizados ${ }^{2}$, para lo cual existen dos maneras comunes: la primera toma en consideración un rango de 0 a 1 con números reales y la segunda utiliza un rango de 0 al 255 con número enteros; en ambos casos los valores más altos son los más adecuados. Para el desarrollo de esta investigación se ha elegido la normalización de 0 a 255, debido a que en términos técnicos estos archivos son menores, cubren un rango amplio de valores y los procesos requieren menor capacidad de las computadoras (Gómez \& Barredo, 2005:171).

Existen diversos procedimientos para la modelación de los criterios, uno muy empleado es la matriz de comparación por pares de Saaty, en donde se comparan cada uno de los factores contra

\footnotetext{
${ }^{2}$ La normalización es un proceso estadístico que regularmente llamamos estandarización; las formas más comunes son a partir de porcentajes, tasas, densidades, promedios e índices, entre otros. La característica principal es que deben estar medidos en la misma escala, es decir, los valores máximos y mínimos son los mismos en la transformación de todos los factores, por ejemplo, de 0 al (utilizando valores reales), de 0 a 100 , de 0 a 255 , etc.
} 


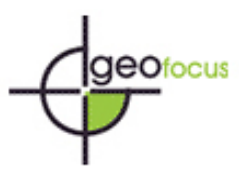

Santana Castañeda, G., Aguilar Martínez, A.G. (2020). Sitios candidatos para nuevos servicios médicos utilizando técnicas de evaluación multicriterio, en la Zona Metropolitana de Toluca, México. GeoFocus, Revista Internacional de Ciencia y Tecnología de la Información Geográfica, 26, 139-162. http://dx.doi.org/10.21138/GF.602

todos los demás y se le dan valores llamados “juicios de valor", estos valores van desde 1/9 (valores fraccionados) hasta 9 (valores enteros).

Cuando se asignan valores fraccionados $(1 / 9 \ldots 1 / 2)$ el factor que se encuentra en la primera columna es menos importante que el factor en el primer renglón, en dónde el valor numérico sugiere la intensidad de esta condición. Por consiguiente, se si asignan valores enteros (2...9) entonces sugiere lo contrario, y los valores que coinciden en diagonal se les asigna un valor de 1, debido a que ninguno es más importante que el otro, pues es el mismo factor.

La asignación de estos valores se realiza mediante una matriz cuadrada cuya cantidad de filas y columnas está dada por el número de factores a ponderar, la comparación siempre se realiza con un par de variables (las que se encuentran en los renglones contra las que se encuentran en las columnas, por ejemplo, $\mathrm{P}_{\mathrm{ba}}$ ), tal como se muestra en la tabla 1 .

Tabla 1. Matriz de comparación por pares de Saaty

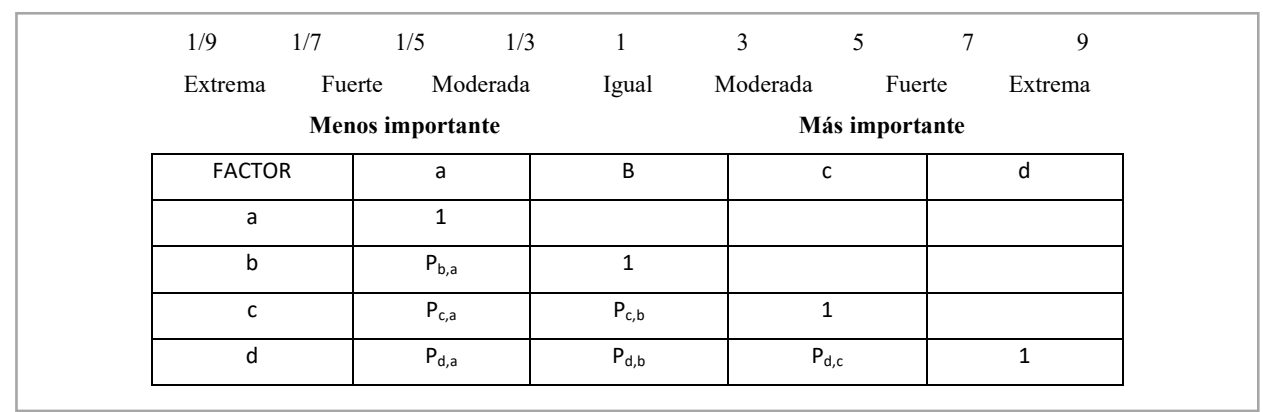

Fuente: Elaboración propia con base en Gómez, M., y Barredo, J. I., 2005.

Después de asignar los juicios de valor se deben calcular los pesos para cada uno de los factores tal como se muestra en la tabla 2. En primer lugar, se complementa la matriz (se muestran estos valores en color gris) y después se suman los valores de cada columna. En segundo lugar, se divide cada valor con referencia al total de cada columna y al final se suma cada renglón para posteriormente dividir el resultado entre el número total de factores, con lo que se obtiene la ponderación de cada factor.

Estas ponderaciones siempre deben sumar 1 y deben encontrarse en términos aritméticos precisos, lo que se mide con el índice de consistencia: cuando este valor se encuentra más cercano a 0 , entonces la consistencia o la lógica con la que fueron asignados estos juicios de valor tiende a ser perfecta.

La asignación de los pesos es un proceso importante pues de ello depende en gran medida el resultado, por lo que el decisor puede establecer estos pesos considerando la relevancia de los factores, realizando una revisión bibliográfica, una consulta a experto (s), tomadores de decisiones, gestores, grupos de interés, etc.

El número sugerido de factores para realizar la comparación es de, como máximo, 7, ya que según Pomerol y Barba (1997), citado por Gómez y Barredo (2005:50) "La carga cognitiva para el decisor sería tan fuerte que no se puede esperar recopilar información estable y significativa". 
Santana Castañeda, G., Aguilar Martínez, A.G. (2020). Sitios candidatos para nuevos servicios médicos utilizando técnicas de evaluación multicriterio, en la Zona Metropolitana de Toluca, México. GeoFocus, Revista Internacional de Ciencia y Tecnología de la Información Geográfica, 26, 139-162. http://dx.doi.org/10.21138/GF.602

Tabla 2. Proceso para la matriz de comparación por pares de Saaty.

\begin{tabular}{|l|l|l|l|}
\hline \multicolumn{4}{|l|}{ Paso 1. Asignación de "juicios de valor" } \\
\hline Factores & A & b & C \\
\hline a & 1 & 3 & $1 / 2$ \\
\hline b & $1 / 3$ & 1 & $1 / 5$ \\
\hline c & 2 & 5 & 1 \\
\hline
\end{tabular}

Paso 2. Calcular los pesos en valores decimales y suma de columnas

\begin{tabular}{|l|l|l|l|}
\hline Factores & A & b & c \\
\hline a & 1 & 3 & $(1 / 2=) 0.5$ \\
\hline b & $(1 / 3=) 0.33$ & 1 & $(1 / 5=) 0.2$ \\
\hline c & 2 & 5 & 1 \\
\hline$\sum$ & 3.33 & 9.00 & 1.70 \\
\hline
\end{tabular}

Paso 3. Suma de renglones y obtención de pesos.

\begin{tabular}{|l|l|l|l|l|l|}
\hline Factores & $\mathrm{a}$ & $\mathrm{b}$ & $\mathrm{c}$ & $\sum$ & Pesos \\
\hline $\mathrm{a}$ & $(1 / 3.33=) 0.3$ & $(3 / 9=) 0.33$ & $(0.5 / 1.7=) 0.29$ & 0.93 & $(0.93 / 3=) \mathbf{0 . 3 0 9 1 5}$ \\
\hline $\mathrm{b}$ & $(0.33 / 3.33=) 0.1$ & $(1 / 9=) 0.11$ & $(0.2 / 1.7=) 0.12$ & 0.33 & $(0.33 / 3=) \mathbf{0 . 1 0 9 5 8 6}$ \\
\hline $\mathrm{c}$ & $(2 / 3.33=) 0.6$ & $(5 / 9=) 0.56$ & $(1 / 1.7=) 0.59$ & 1.74 & $(1.74 / 3=) \mathbf{0 . 5 8 1 2 6 4}$ \\
\hline \multicolumn{7}{|l}{} & $\sum$ & $\mathbf{1}$ \\
\hline
\end{tabular}

Fuente: Elaboración propia con base en Gómez, M., y Barredo, J. I., 2005.

Otro método muy común y sencillo para establecer ponderaciones es el de Malczewski (1999:177) basado en la jerarquización de los diferentes valores para realizar una ponderación. La ecuación es la siguiente:

Ecuación 1. Jerarquías de Malczewski

Dónde $\mathrm{r}=$ ranking y $\mathrm{j}=$ criterio.

$$
P_{j}=\left(1 / r_{j}\right) / \Sigma\left(1 / r_{j}\right)
$$

Otros métodos para establecer ponderaciones a los factores son: el método de las utilidades relativas, que consiste básicamente en que a partir de las estimaciones provisionales se vayan modificando con el objetivo de mejorar su consistencia interna por medio de comparaciones binarias de subgrupos de criterios. Otro método es mediante comparaciones binarias de los criterios (como AHP o Analytic Hierarchy Process de Saaty). Otro es el método Delphi de consenso que combinado o no, con los métodos anteriores, se desarrollan a partir de grupos de decisores.

Para el desarrollo del presente documento se utilizaron dos métodos: primero el de Saaty, seguido por el de Malczewski. 


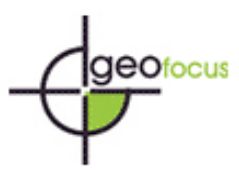

Santana Castañeda, G., Aguilar Martínez, A.G. (2020). Sitios candidatos para nuevos servicios médicos utilizando técnicas de evaluación multicriterio, en la Zona Metropolitana de Toluca, México. GeoFocus, Revista Internacional de Ciencia y Tecnología de la Información Geográfica, 26, 139-162. http://dx.doi.org/10.21138/GF.602

\section{d) Reglas de decisión}

La regla de decisión de la combinación de criterios es el mecanismo por el que se combinan los factores para producir una única valoración de cada alternativa, lo que permite seleccionar una de ellas como la más adecuada.

Los tipos de reglas de decisión son:

a) Combinación booleana, donde todos los criterios son restricciones (1 y 0 ). Para combinar variables binarias se pueden usar operadores booleanos:

Y lógica, cuando un punto es apto o verdadero únicamente en el caso en que todos los valores de los criterios en ese lugar tengan valores de verdad (1). Es un operador muy restrictivo por que la unidad espacial debe cumplir con el valor de 1 en todos sus criterios.

O lógica, cuando un punto es apto o verdadero en el caso de que al menos uno de los valores en los criterios tenga valores verdaderos o aptos. Es un operador no restrictivo, muy liberal y poco conservador.

b) Ordinales, como el método de precedencias en donde los factores están medidos en escala ordinal (importancia) y requiere realizar comparaciones de dos en dos entre todas las alternativas, por lo que es poco viable para utilizarlo con los SIG.

c) Cuantitativos, incluye factores medidos en la escala de intervalos o de razón, como la suma lineal ponderada (SLP) o análisis del punto ideal.

Para el desarrollo de esta investigación se toma en cuenta una consulta a expertos y se utilizaran combinaciones booleanas con Y lógica.

Para contextualizar la temática que se aborda, a continuación, se muestra un diagnóstico sobre la cobertura de los servicios médicos y las áreas de oportunidad que dan lugar al presente trabajo.

\section{2. Área de estudio}

El área de estudio es la Zona Metropolitana de Toluca (ZMT) está integrada por 15 municipios los cuales son: Almoloya de Juárez, Calimaya, Chapultepec, Lerma, Metepec, Mexicaltzingo, Ocoyoacac, Otzolotepec, Rayón, San Antonio la Isla, San Mateo Atenco, Toluca, Xonacatlán, Zinacantepec y Temoaya (CONAPO, SEDESOL e INEGI, 2012:104).

Según el censo de población y vivienda de 2010 realizado por el INEGI, la población en la ZMT es de 1,936,126 habitantes, de los cuales el $48.7 \%(942,165)$ son del sexo masculino y el $51.3 \%(993,961)$ son mujeres. La tasa de crecimiento de la región en el periodo de 2005 a 2010 fue de 2.5 superior al del valor estatal que fue de 1.6, el municipio que registró el mayor crecimiento fue San Antonio la Isla con 14.4, le sigue Chapultepec con 8.0 y Lerma con 5.0, debido principalmente a la creación de fraccionamientos habitacionales. 
Santana Castañeda, G., Aguilar Martínez, A.G. (2020). Sitios candidatos para nuevos servicios médicos utilizando técnicas de evaluación multicriterio, en la Zona Metropolitana de Toluca, México. GeoFocus, Revista Internacional de Ciencia y Tecnología de la Información Geográfica, 26, 139-162. http://dx.doi.org/10.21138/GF.602

Para el 2015 la población en la ZMT es de 2,116,506 habitantes se mantiene el porcentaje entre hombres $(48.4 \%)$ y mujeres $(51.6 \%)$. La tasa de crecimiento fue de 1.8 menor al del periodo anterior, pero superior al del estatal que fue de 1.3.

La ZMT incluye 102 localidades urbanas (17.9\% del total de localidades en la ZMT) que tienen una población de 1,537,001 (79.4\%) y 466 localidades rurales $(82.04 \%$ del total) las cuales tienen una población de 399,125 (20.6\%) habitantes al año 2010 (ver figura 4).

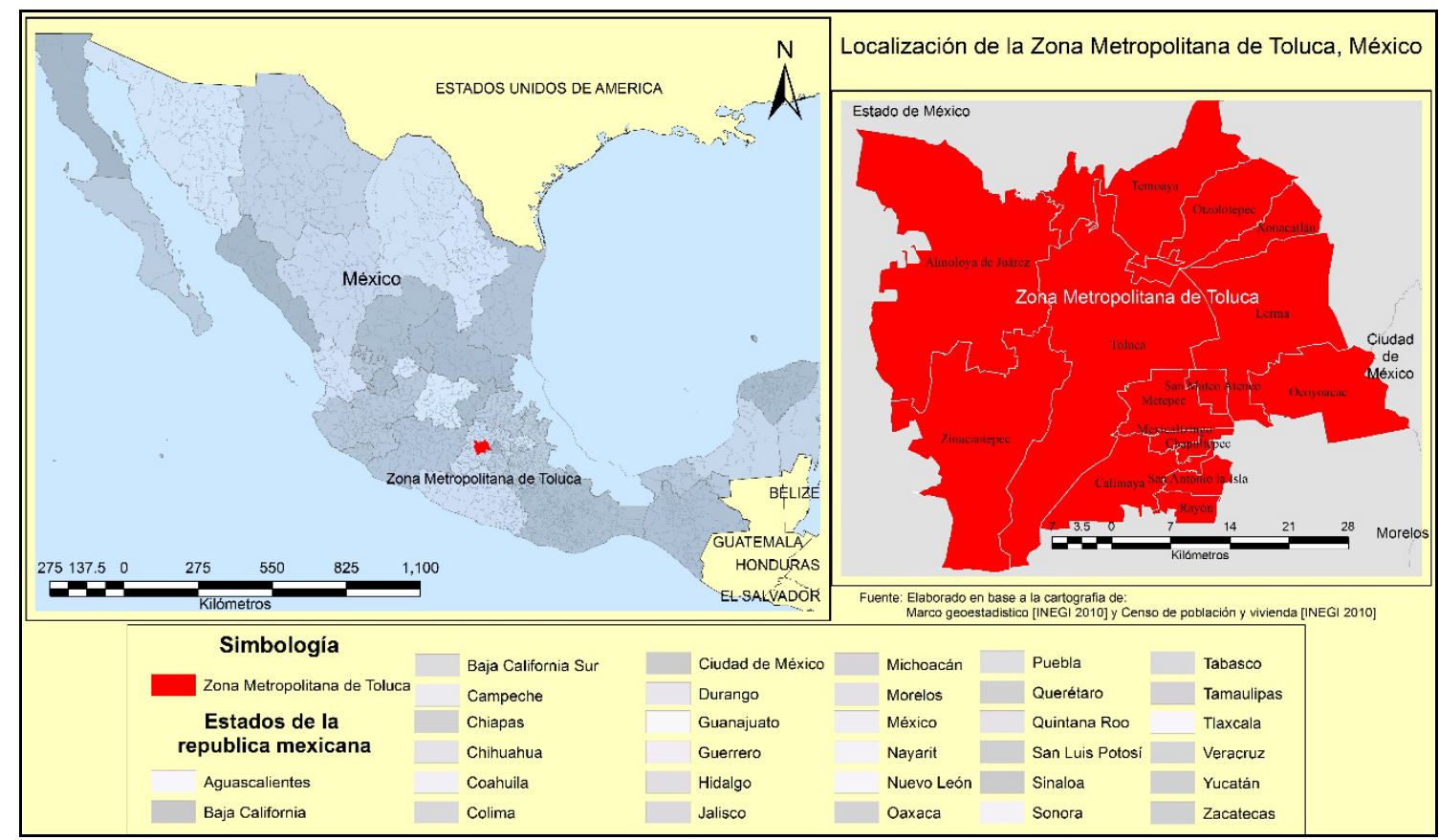

Figura 4. Localización de la Zona Metropolitana de Toluca, México

Fte: Elaboración propia con base en el Marco geoestadístico municipal, INEGI 2010.

En México, el sistema nacional de salud público está caracterizado por la segmentación y fragmentación de sus instituciones, que coexisten actuando de manera independiente y desarticulada, condicionados por la disponibilidad de recursos por parte del Estado y la capacidad organizativa del propio sistema de salud, padeciendo de duplicidad de información y desperdicio de recursos (PAHO, 2017) y cuyo acceso está en función de la inserción laboral, además existen tres niveles de atención: el primario, que es el nivel de atención más básico, el segundo corresponde a los hospitales generales y el terciario a hospitales especializados.

Dentro de las principales instituciones se encuentra la Secretaria de Salud (SSA), el Instituto Mexicano del Seguro Social (IMS), Instituto de Seguridad y Servicios Sociales para los Trabajadores del Estado (ISSSTE), el Sistema Nacional para el Desarrollo Integral de la Familia (SNDIF), Hospitales Universitarios (HU), la Cruz Roja Mexicana (CRM) y Servicios Médicos 
Santana Castañeda, G., Aguilar Martínez, A.G. (2020). Sitios candidatos para nuevos servicios médicos utilizando técnicas de evaluación multicriterio, en la Zona Metropolitana de Toluca, México. GeoFocus, Revista Internacional de Ciencia y Tecnología de la Información Geográfica, 26, 139-162. http://dx.doi.org/10.21138/GF.602

Estatales (SME), Secretaría de Comunicaciones y Transporte (SCT), Petróleos mexicanos (Pemex), Defensa, Marina, seguros estatales, entre otros.

La SSA es una de las secretarías de estado que integran el denominado gabinete legal del presidente de México, es la encargada de diseñar, ejecutar y coordinar las políticas públicas en materia de servicios sanitarios, además de recabar toda la información sobre el tema.

Los servicios médicos que se analizan en el presente documento pertenecen a la Secretaria de Salud, debido principalmente a que están dirigidos a toda la población incluso la que no cuenta con derechohabiencia, además el nivel de atención que se analiza es el primero, de primer contacto o unidades de consulta externa3, que para el año 2016 en la Zona Metropolitana de Toluca son 164.

En México para el 2016, los servicios médicos de primer nivel privados ocupan el $3 \%$ y los servicios públicos ocupan el $97 \%$, de ellos, la Secretaria de Salud tiene el $69.9 \%$ del total de unidades de consulta externa (UCE).

Estas unidades de consulta externa no son del mismo tamaño ni ofrecen los mismos servicios, existe una clasificación llamada tipología. Esta tipología está dividida según su orientación, que puede ser hacía localidades rurales (con cuatro tipos) y si es para localidades urbanas existen 15, la diferencia es la infraestructura y dotación.

Por ejemplo, los centros de salud rurales se ubican en localidades que tienen población dispersa cuentan con un consultorio médico y su respectivo núcleo primario, este núcleo incluye una sala de espera, baño de usuarios, y habitación para el médico con cocineta y baño. En este tipo de unidad se proporciona atención clínica básica como promoción de la salud, saneamiento ambiental, diagnóstico comunitario temprano de enfermedades, detección y control de riesgos.

Los centros de salud para "población rural no dispersa y urbana" se clasifican entre dos y 12 consultorios, así como sus núcleos primarios correspondientes a la sala de espera, sala de expulsión y consultorio de estomatología. Según su tamaño, aumentan los servicios de telecomunicación (radio, telefonía, sistemas informáticos para gestión de pacientes y telemedicina), farmacia, almacén, oficina para director y oficina para enfermería. En estas unidades se proporciona atención clínica integral, atención de urgencias y cuando se ubican a menos de 30 minutos de un hospital de referencia, atención del parto eutócico, suministran también servicios básicos de salud a la comunidad (promoción de la salud, saneamiento ambiental, diagnóstico comunitario temprano de enfermedades, detección y control de riesgos); cuando cuentan con más de tres núcleos básicos ofrecen servicios matutinos y vespertinos los 365 días del año y atención de urgencias las 24 horas del día (Modelo integrador de atención a la salud, 2006). Ver figura 5.

\footnotetext{
3 Las unidades de consulta externa son aquellos establecimientos donde se otorgan servicios de tipo ambulatorio, y por lo tanto no hay servicios de urgencias puesto que no existe la infraestructura necesaria (por ejemplo: partos o lesiones que requieran cirugía, etc.). La consulta externa se clasifica como general cuando es otorgada por un médico general o de familia, mientras que se clasifica como de especialidad cuando es otorgada por un médico especialista, ya sea de alguna de las especialidades o de subespecialidad. Sistema Nacional de Información en Salud (SINAIS).
} 


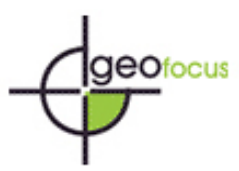

Santana Castañeda, G., Aguilar Martínez, A.G. (2020). Sitios candidatos para nuevos servicios médicos utilizando técnicas de evaluación multicriterio, en la Zona Metropolitana de Toluca, México. GeoFocus, Revista Internacional de Ciencia y Tecnología de la Información Geográfica, 26, 139-162. http://dx.doi.org/10.21138/GF.602

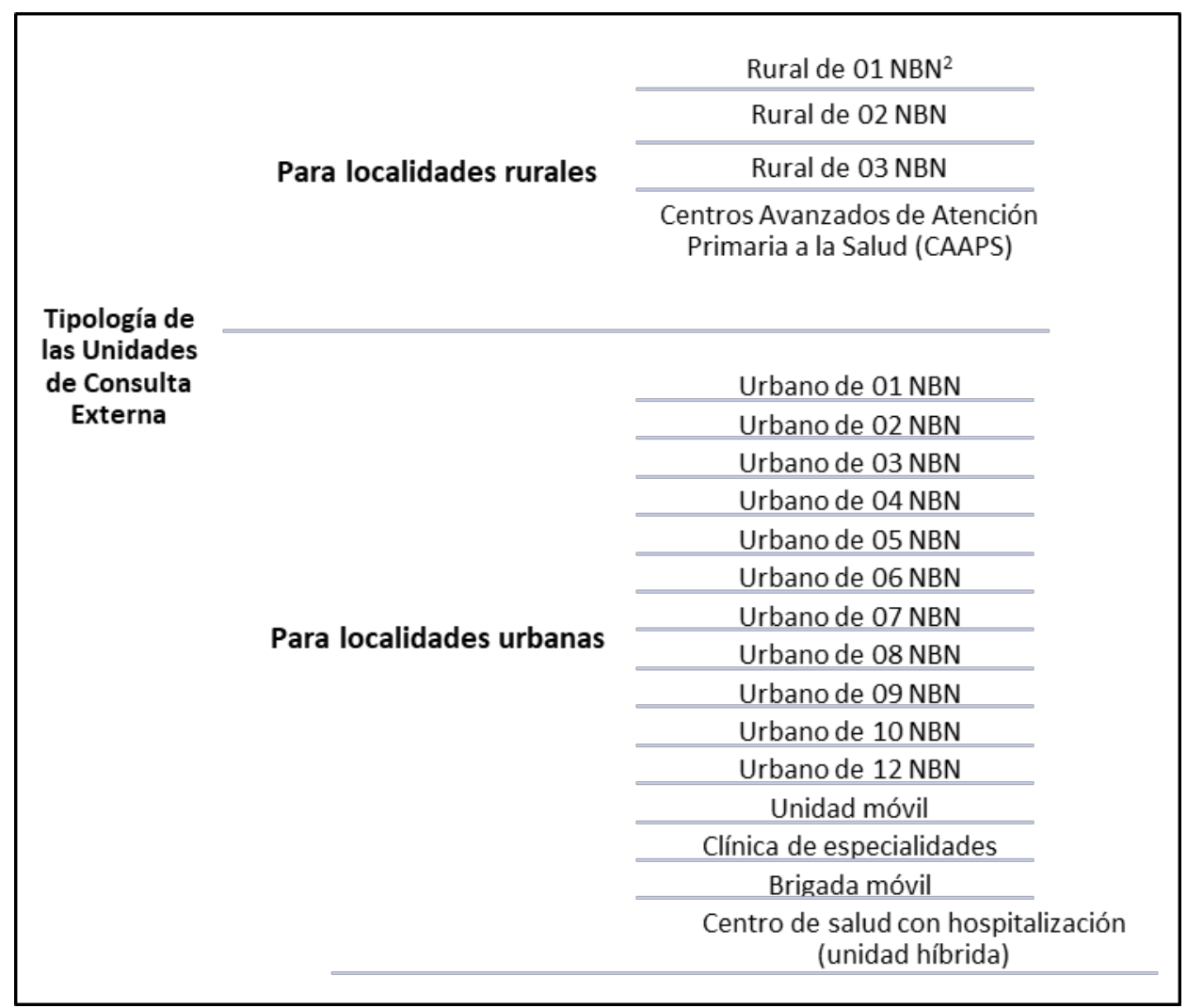

Figura 5. Tipología de las unidades de consulta externa

Fte: Elaboración propia con base en el Modelo de atención a la salud para población abierta, 1995; Sistema normativo de equipamiento urbano, 1999 y Modelo integrador de atención a la salud, 2006

El objetivo de este trabajo es examinar las posibilidades que tienen las técnicas de evaluación multicriterio para evaluar una serie de alternativas que satisfagan, en este caso un objetivo, considerando el uso de múltiples criterios, para determinar zonas con mayor aptitud territorial para ubicar nuevos servicios médicos de primer nivel.

La OMS (2008) menciona que la atención primaria de salud se presentó como un conjunto de valores, principios y enfoques destinados a mejorar el grado de salud de las poblaciones desfavorecida, también menciona que los sistemas de salud son injustos, inconexos, ineficientes y menos eficaces de lo que podrían ser. Además, sin una reorientación sustancial es probable que los actuales sistemas de salud se vean desbordados por los crecientes desafíos del envejecimiento de la población, las pandemias de enfermedades crónicas, las nuevas enfermedades emergentes, como el SRAS, y las repercusiones del cambio climático, por lo que es de suma importancia que los servicios médicos tengan cubierta a toda la población, incluso y con mayor importancia a la población en desventaja. 
Santana Castañeda, G., Aguilar Martínez, A.G. (2020). Sitios candidatos para nuevos servicios médicos utilizando técnicas de evaluación multicriterio, en la Zona Metropolitana de Toluca, México. GeoFocus, Revista Internacional de Ciencia y Tecnología de la Información Geográfica, 26, 139-162. http://dx.doi.org/10.21138/GF.602

\section{Metodología}

Las fuentes de información son la Secretaria de Salud (SSA), a través de la Dirección General de Información en Salud (DGIS) y el Instituto Nacional de Estadística y Geografía (INEGI).

En primer lugar, se realizó un ejercicio básico, utilizando un modelo de asignación, entre la oferta (las UCE) y a demanda (la población en la Zona Metropolitana de Toluca), tomando en consideración los parámetros que especifica el modelo integrador de atención a la salud, MIDAS por sus siglas (SSA, 2006) para obtener las áreas desprovistas de este servicio médico. Y, en segundo término, se obtendrán las áreas potenciales para la localización de los nuevos servicios, las técnicas utilizadas son la comparación por pares de Saaty y el Jerárquico de Malczewski.

Para realizar el modelo de asignación, se toma en consideración la demanda, que es el total de población que habita la Zona Metropolitana de Toluca, también la oferta que son las 1654 unidades de consulta externa, esta asignación va a tomar en cuenta la normatividad con respecto a la tipología de cada una de las unidades de consulta externa referidas del modelo integrador de atención a la salud publicado en 2006, en dónde se menciona que debe haber de 1 a 2 médicos por 1,000 habitantes; de 1 a 2 consultorios por 1,000 habitantes; de 2 a 3 licenciadas en enfermería por médico y de 1 a 2 camas por 1,000 habitantes, ver figura 6 .

Este modelo muestra como resultado una distancia lineal mínima de 62 metros y una máxima de 4,142 metros, esto significa que 818 (63\%) puntos de población no fueron asignados a ningún servicio médico.

El $25 \%$ de los valores más altos de cobertura se localizan en 13 UCE que se encuentran en los municipios de Almoloya, Zinacantepec, Toluca, Rayón, San Antonio la Isla, Chapultepec, Metepec, San Mateo Atenco y Lerma. El siguiente $25 \%$ lo ocupan 30 UCE distribuidas en los municipios de Almoloya, Zinacantepec, Toluca, Temoaya, Otzolotepec, Xonacatlán, Lerma y Oriente del municipio de Ocoyoacac. En términos generales, cuando se considera la normatividad un gran porcentaje de población se queda sin algún tipo de asignación, fundamentalmente en las áreas conurbadas de los municipios. 


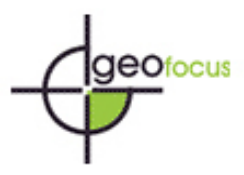

Santana Castañeda, G., Aguilar Martínez, A.G. (2020). Sitios candidatos para nuevos servicios médicos utilizando técnicas de evaluación multicriterio, en la Zona Metropolitana de Toluca, México. GeoFocus, Revista Internacional de Ciencia y Tecnología de la Información Geográfica, 26, 139-162. http://dx.doi.org/10.21138/GF.602

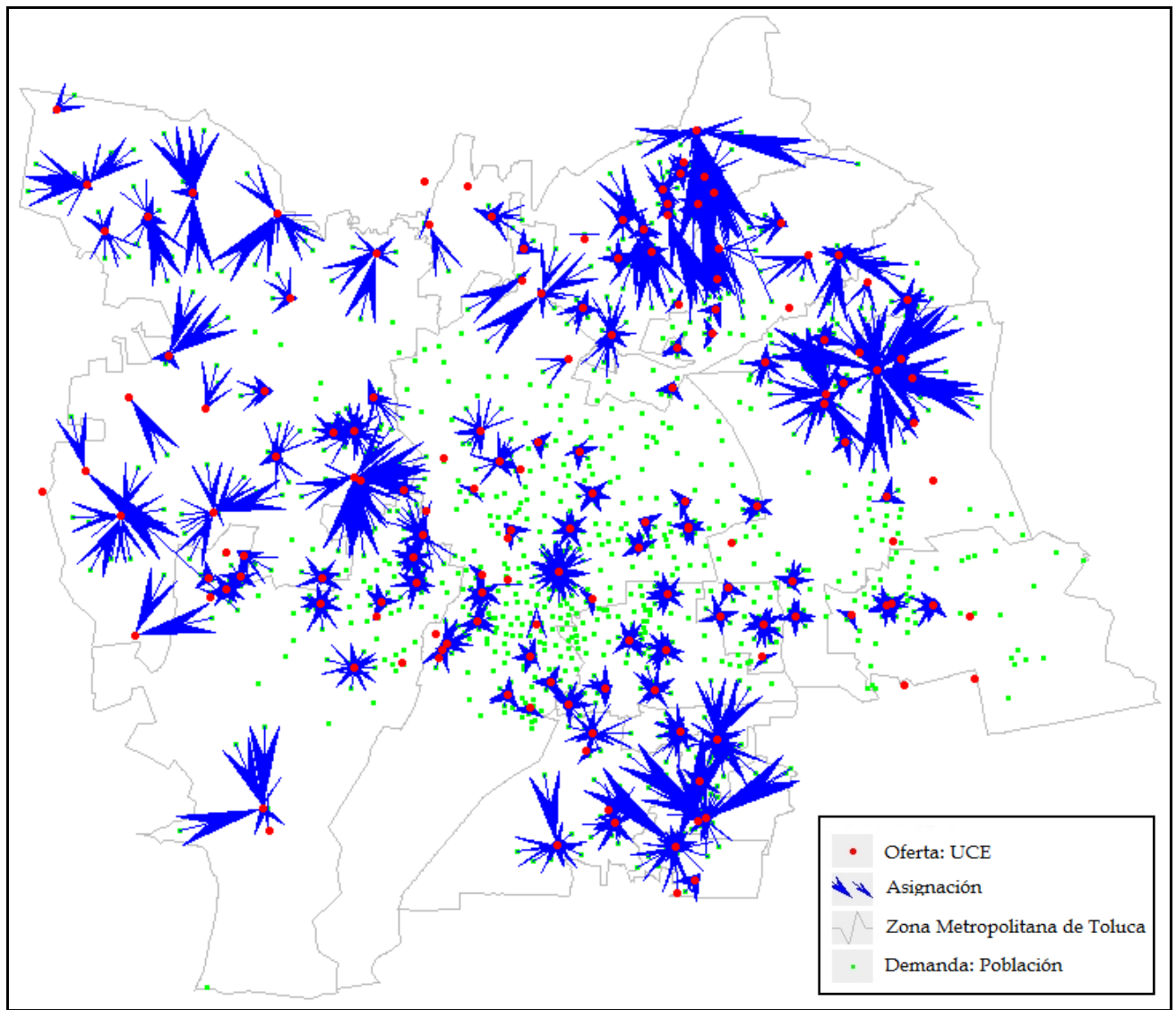

Figura 6. Zona Metropolitana de Toluca, modelo de cobertura tomando en cuenta la tipología de las unidades de consulta externa por número de habitantes

Fte. Elaboración propia con base Flowmap, datos de Dirección General de Información en Salud SINAIS, 2016 y Censo de población y vivienda, INEGI 2010.

Los patrones de distribución espacial de las características del equipamiento de estos servicios proporcionan a la población diferentes niveles de accesibilidad y por lo tanto irregulares oportunidades para su uso y disfrute, dando superiores condiciones en la parte centro y menores en la parte aledaña.

El análisis de las imperfecciones en el suministro de estos servicios resulta un asunto de permanente interés a causa de las variaciones en el acceso espacial y las desigualdades que genera, de tal modo que se observan áreas de oportunidad, en dónde es necesario el servicio médico para mejorar la calidad de vida de toda la población en la Zona Metropolitana de Toluca, es por eso que en el presente documento se proponen sitios con mayor aptitud, mediante técnicas de evaluación multicriterio para que se puedan construir nuevos servicios médicos de primer nivel. Para llegar a ello se presenta el siguiente apartado que aborda el método utilizado. 
Santana Castañeda, G., Aguilar Martínez, A.G. (2020). Sitios candidatos para nuevos servicios médicos utilizando técnicas de evaluación multicriterio, en la Zona Metropolitana de Toluca, México. GeoFocus, Revista Internacional de Ciencia y Tecnología de la Información Geográfica, 26, 139-162. http://dx.doi.org/10.21138/GF.602

\section{Sitios con aptitud locacional para nuevos servicios médicos}

El modelo de atención a la salud para población abierta publicado en 1995 señala que se deben tomar en cuenta diferentes factores para ampliar la cobertura de los servicios de salud, estos son: el número y tamaño de las localidades, grado de dispersión, concentración y densidad geográfica, condiciones étnicas y socioculturales, situación epidemiológica, disponibilidad de medios, así como vías de comunicación con tiempos de traslado definidos de acuerdo a las condiciones locales y el acceso a los servicios, estos criterios se toman en cuenta más adelante en la selección de criterios.

\section{a) Selección de criterios}

La selección de criterios es el paso inicial del proceso de evaluación multicriterio, pues es considerado el punto de referencia para la toma de decisiones, siempre se toma en cuenta el objetivo principal de la investigación. Se seleccionaron variables consideradas de aptitud para determinar sitios candidatos para la localización de nuevos establecimientos de salud pública obtenidos de la normatividad vigente en México: el sistema normativo de equipamiento urbano de la Secretaría de Desarrollo Social (999:20), el modelo de atención a la salud para población abierta (1995:20), el modelo integrador de atención a la salud (2006:40), así como la revisión bibliográfica bajo la teoría del bienestar para proponer estrategias enfocadas a beneficiar a la población que se encuentra en desventaja.

El modelo de evaluación multicriterio que se desarrolló para obtener los sitios candidatos para nuevas unidades de consulta externa está basado en tres conjuntos de factores y tres restricciones.

El primer conjunto de factores denominado de servicio está integrado por un sólo factor relacionado con la lejanía a las unidades médicas existentes, el segundo grupo incluye dos factores con características físicas: la cercanía a las vías de comunicación y la pendiente, y para el tercer grupo perteneciente a las necesidades de la población se utilizaron los siguientes factores: grado promedio de escolaridad, población sin derechohabiencia, población con limitación en la actividad, índice de dependencia, viviendas particulares habitadas que no disponen de refrigerador y tasa de mortalidad.

Para obtener las ponderaciones de los factores en el tercer grupo se utilizó el método de Saaty, (ver figura 1) y para obtener la ponderación de los grupos se utilizó el método de Malczewski, (ver ecuación 1), esto se puede ver gráficamente en la figura 6. 
Santana Castañeda, G., Aguilar Martínez, A.G. (2020). Sitios candidatos para nuevos servicios médicos utilizando técnicas de evaluación multicriterio, en la Zona Metropolitana de Toluca, México. GeoFocus, Revista Internacional de Ciencia y Tecnología de la Información Geográfica, 26, 139-162. http://dx.doi.org/10.21138/GF.602

\section{MODELO DE EVALUACIÓN MULTICRITERIO}

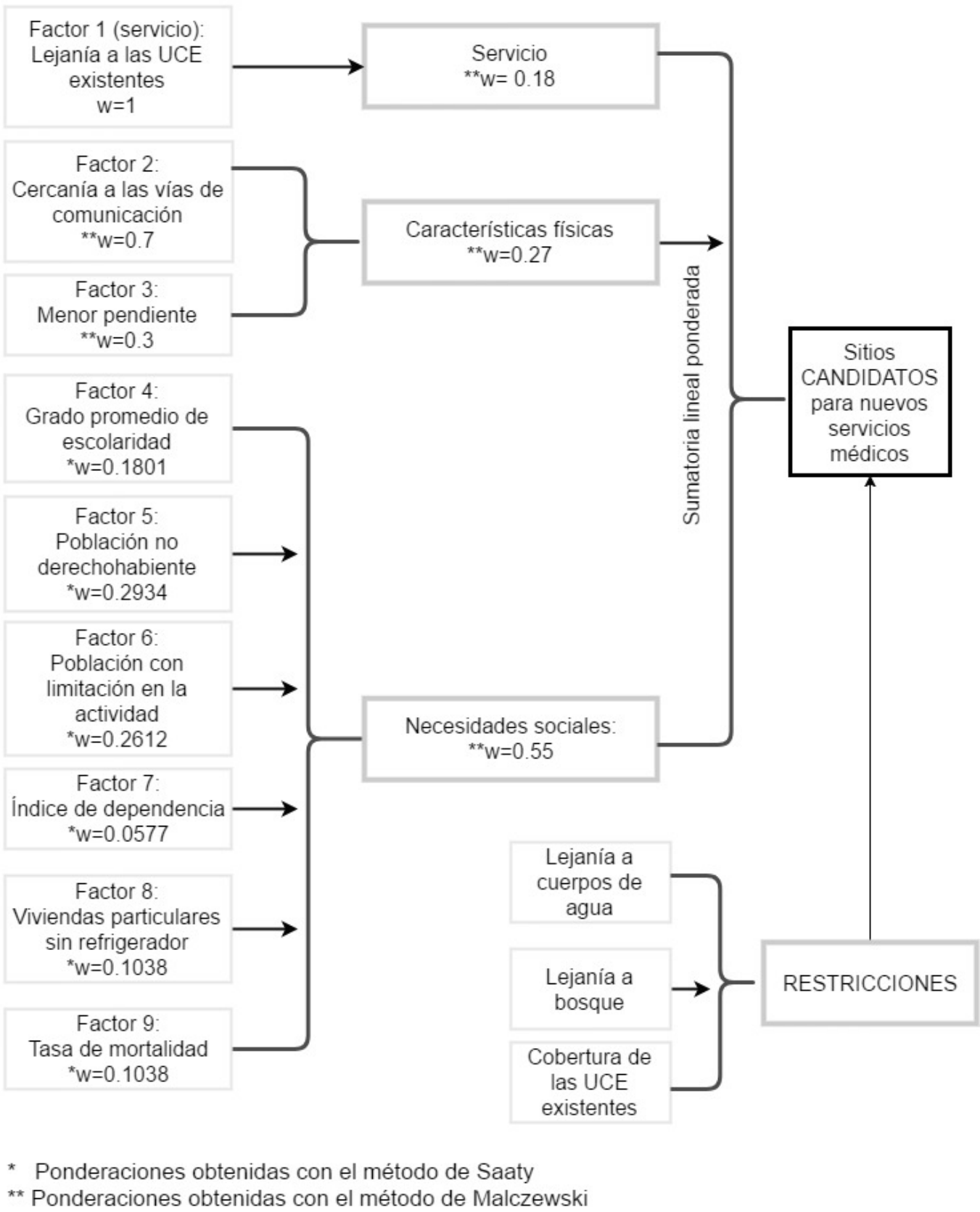

Figura 3. Esquema metodológico: Sitios candidatos para nuevos servicios médicos en la Zona Metropolitana de Toluca

Fte: Elaboración propia, 2016. 
Santana Castañeda, G., Aguilar Martínez, A.G. (2020). Sitios candidatos para nuevos servicios médicos utilizando técnicas de evaluación multicriterio, en la Zona Metropolitana de Toluca, México. GeoFocus, Revista Internacional de Ciencia y Tecnología de la Información Geográfica, 26, 139-162. http://dx.doi.org/10.21138/GF.602

\section{b) Normalización de factores}

La normalización se realizó utilizando valores en un rango de 0 al 255 (con números enteros) donde los valores más altos corresponden a los más adecuados utilizando una función de tipo lineal, además según correspondan las aptitudes de cada uno de los factores se utiliza una función de incremento o decremento monótono.

El primer factor que corresponde a la localización y lejanía de los centros de salud parte de una imagen ${ }^{4}$ que contiene las unidades de consulta externa para hacer una operación de distancia y se normaliza utilizando el módulo llamado fuzzy, se utilizó una función de tipo lineal, incremento monótono, con valores de 0 a 255. La ponderación final es de 0.18 .

Para el segundo conjunto de factores, se toma en consideración dos imágenes de entrada que son las vías de comunicación y la altitud. A la imagen de vías de comunicación se hace una operación de distancia y una normalización con una función de tipo lineal, decremento monótono con valores de 0 a 255 , mientras que la imagen de altitud se transformó en pendiente y se normalizo con una función de tipo lineal, decremento monótono, con valores de 0-255. Las ponderaciones finales son: 0.189 a la cercanía con las vías de comunicación y 0.189 a la pendiente.

Para el tercer factor se toma en cuenta la demanda potencial, que incluye variables socioeconómicas y epidemiológicas; estas son:

- Grado promedio de escolaridad (Variable de escolaridad)

- Población no derechohabiente (Variable de salud)

- Población con limitación en la actividad (Variable de población)

- Índice de dependencia (Variable de población)

- Viviendas particulares habitadas que no disponen de refrigerador (Variable de vivienda)

- Mortalidad (Variable epidemiológica) No se considera a la morbilidad, debido a que los datos que se pueden georreferenciar se asocian a un centro de salud existente, y lo que se quiere saber es la demanda potencial de los sitios en donde no hay servicios médicos.

En el presente estudio la metodología emplea principios teóricos basados en justicia espacial y distributiva, así como en la teoría del bienestar, debido a ello, los procedimientos que aquí se plantean no tratan por igual a toda la población, lo que implica, que existe una diferenciación relacionada a la necesidad existente entre los grupos sociales apoyada en diversos indicadores socioeconómicos como variable (s) que condicionan estas necesidades.

La teoría del bienestar propuesta por Smith (1977:121) para servicios públicos señala que debe haber una equidad vertical, un trato desigual para necesidades desiguales esta forma de equidad se enfoca en las desigualdades o brechas injustas que existen entre personas o grupos, por lo tanto, se

\footnotetext{
${ }^{4}$ La evaluación multicriterio se desarrolló en el software Idrisi. Los datos de entrada son imágenes de tipo raster, importadas de un archivo *.shp. Todas las imágenes utilizadas tienen 7200 columnas y 6500 renglones, la resolución del pixel es de 10 x 10 metros, y el sistema de referencia es cónica conforme de Lambert.
} 


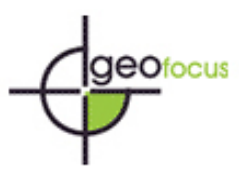

Santana Castañeda, G., Aguilar Martínez, A.G. (2020). Sitios candidatos para nuevos servicios médicos utilizando técnicas de evaluación multicriterio, en la Zona Metropolitana de Toluca, México. GeoFocus, Revista Internacional de Ciencia y Tecnología de la Información Geográfica, 26, 139-162. http://dx.doi.org/10.21138/GF.602

favorecerán las peores condiciones existentes en la Zona Metropolitana de Toluca para la selección de la mejor aptitud locacional.

Para la implementación de este factor se realizó la unión de dos imágenes que contienen los valores de las variables expuestas anteriormente, la primera referida a las localidades rurales y la segunda a las localidades urbanas con un nivel de desagregación de ageb ${ }^{5}$; después se realiza una estandarización con valores de 0 a 255 y una función de tipo lineal, diferenciando entre las variables de incremento y decremento monótono, es decir: el grado promedio de escolaridad es de decremento, por el contrario, la no derechohabiencia, población con limitación, índice de dependencia, viviendas particulares habitadas que no disponen de refrigerador y tasa de mortalidad general son de incremento monótono.

\section{c) Restricciones}

Para este estudio las restricciones son tres: no sobre cuerpos de agua, ni bosques y tampoco sobre la cobertura de las unidades médicas existentes, para ello, se debe establecer una zona de seguridad en torno a la zona boscosa y a los cuerpos de agua, esta franja no podrá ser utilizada para ningún uso.

La zona de contención de los cuerpos perenes y de los bosques es de 500 metros y los intermitentes son de 100 metros, debido a ello se realizaron dos áreas buffer y se unieron en una sola imagen.

La obtención de la tercera restricción se obtuvo de la figura 5 del presente artículo, en dónde se muestran las áreas de oportunidad para la nueva localización, que son las que no tuvieron ninguna asignación.

\section{Resultados y discusión}

El método de combinación lineal ponderada (WLC) y el Jerárquico de Malczewski, son procedimientos metodológicos de las técnicas de evaluación multicriterio que muestran la aptitud para la localización de nuevos sitios candidatos de diferentes servicios utilizado en este caso para los servicios médicos, además de ello, se ha tomado como base el consenso de la consulta a expertos y se han asignado diferentes ponderaciones para cada factor.

Los valores más altos en las imágenes son los que tienen mayor aptitud, en este caso, para localizar nuevos servicios de salud el mayor valor es 170 y el mínimo de 15 , como se muestra en la figura 7.

\footnotetext{
${ }^{5}$ AGEB, es el área geoestadística básica urbana, cuya extensión territorial es ocupada por un conjunto de manzanas que generalmente son de 1 a 50 delimitadas por calles, avenidas, andadores o cualquier otro aspecto fácil de identificar en el terreno, y cuyo uso de suelo sea primordialmente habitacional, industrial, de servicio y comercial y sólo se asigna al interior de las localidades urbanas (INEGI, 2010:2)
} 


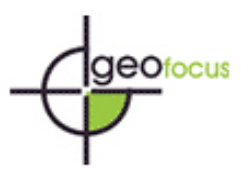

Santana Castañeda, G., Aguilar Martínez, A.G. (2020). Sitios candidatos para nuevos servicios médicos utilizando técnicas de evaluación multicriterio, en la Zona Metropolitana de Toluca, México. GeoFocus, Revista Internacional de Ciencia y Tecnología de la Información Geográfica, 26, 139-162. http://dx.doi.org/10.21138/GF.602

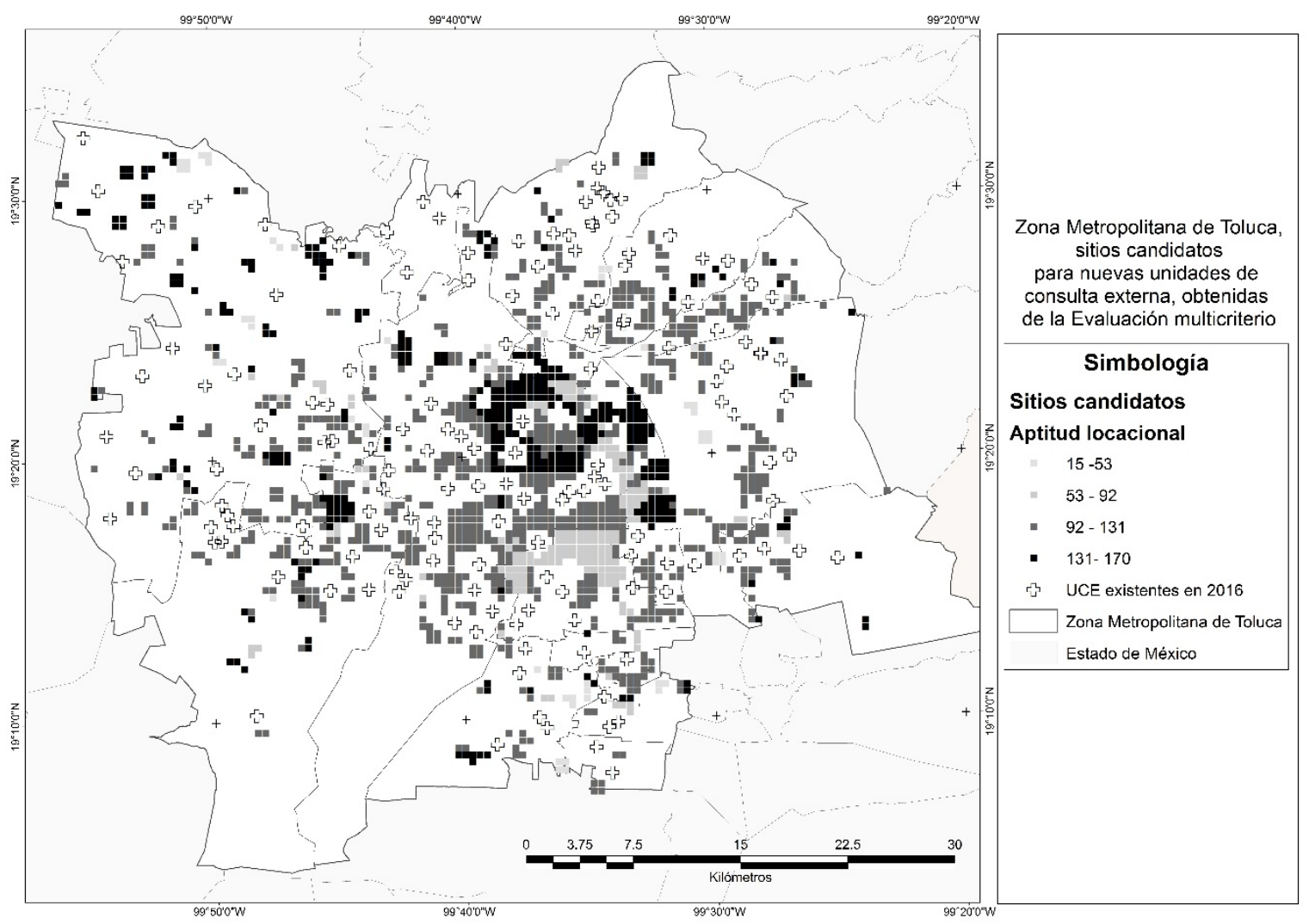

Figura 7. Zona Metropolitana de Toluca, sitios candidatos para nuevas unidades de consulta externa

Fte: Elaboración propia con base en Censo de población y vivienda, INEGI 2010 y Marco geoestadístico, INEGI 2010

Se realiza un corte en el último quintil, que va de 131 a 170 y una depuración cartográfica, considerando que la superficie mínima para construir un nuevo servicio médico es de $1.2 \mathrm{~km}^{2}$, se obtienen 9 áreas agrupadas para sitios candidatos, distribuidos en los municipios de Toluca, Almoloya, Zinacantepec, Calimaya, Otzolotepec y Lerma.

En el municipio de Almoloya de Juárez existen 2 áreas orientados en el noreste y sureste, en el municipio de Calimaya hay un grupo hacía el centro sur, en el municipio de Lerma existen dos conjuntos en el oriente, en el municipio de Otzolotepec hay un grupo en el sur, en el municipio de Toluca hay 3 grupos de gran tamaño ubicados en la parte norte y en el municipio de Zinacantepec hay 2 grupos ubicados en la parte norte.

Por otro lado, los municipios de Temoaya, Ocoyoacac, San Mateo Atenco, San Antonio la Isla, Rayón, Chapultepec, Metepec, Mexicaltzingo y Xonacatlán no presentan sitios candidatos en el último quintil. 


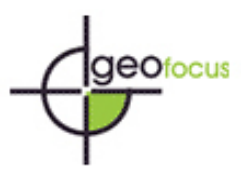

Santana Castañeda, G., Aguilar Martínez, A.G. (2020). Sitios candidatos para nuevos servicios médicos utilizando técnicas de evaluación multicriterio, en la Zona Metropolitana de Toluca, México. GeoFocus, Revista Internacional de Ciencia y Tecnología de la Información Geográfica, 26, 139-162. http://dx.doi.org/10.21138/GF.602

En la figura 8, se reflejan las unidades médicas con símbolos en forma de cruz y los símbolos en forma de cuadro son los sitios candidatos en el último quintil para nuevos servicios médicos con valores de aptitud diferenciada y con una superficie mínima de $1.2 \mathrm{~km}^{2}$.

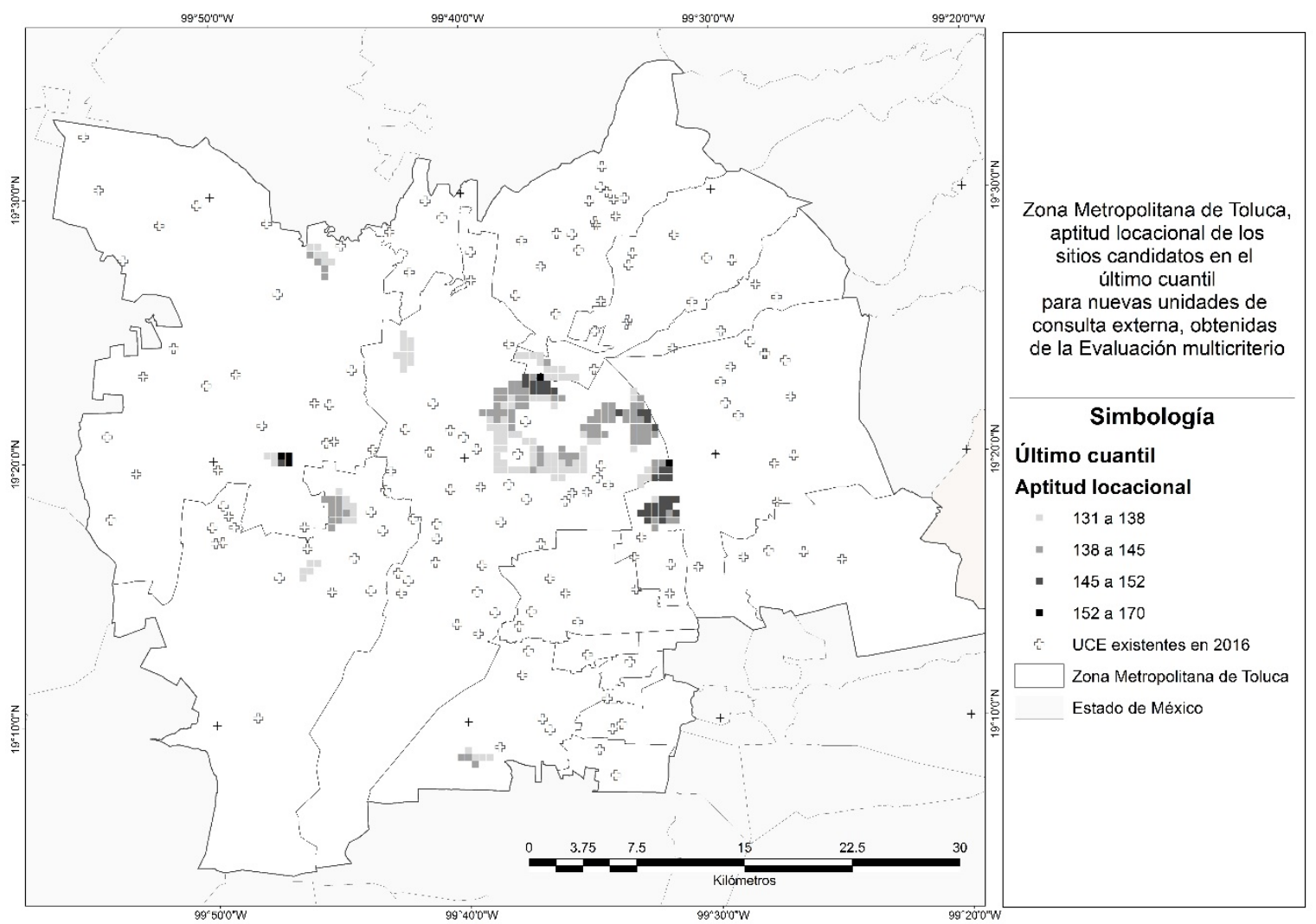

Figura 8. Zona Metropolitana de Toluca, sitios candidatos para nuevas unidades de consulta externa en el cuarto quintil

Fte. Elaboración propia, en base al Censo de población y vivienda (INEGI, 2010) y Marco Geoestadístico (INEGI, 2010)

Este modelo, se ha generado mediante técnicas compensatorias aditivas, eso significa que los factores que se encuentran involucrados se pueden compensar, además de ello, los métodos empleados para definir las ponderaciones, aunque sean diferentes se pueden utilizar para obtener un resultado.

En la figura 6, se muestran las áreas de oportunidad en dónde la población se queda desprovista de los servicios médicos. Esta propuesta toma como base la teoría del bienestar en contraste con las políticas de distribución que actualmente se utilizan para seleccionar las ubicaciones para nuevas infraestructuras, la localización de infraestructura nueva en estos puntos haría que las personas sin derechohabiencia pasen de un modelo segmentado y utilitario a un modelo contractual, en donde cada persona tiene el mismo derecho de la atención y disfrute de 


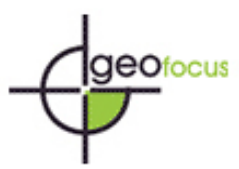

Santana Castañeda, G., Aguilar Martínez, A.G. (2020). Sitios candidatos para nuevos servicios médicos utilizando técnicas de evaluación multicriterio, en la Zona Metropolitana de Toluca, México. GeoFocus, Revista Internacional de Ciencia y Tecnología de la Información Geográfica, 26, 139-162. http://dx.doi.org/10.21138/GF.602

igualdad de oportunidades o justa oportunidad de acceso a los servicios públicos y la atención a los más vulnerables.

El modelo contractual implica considerar los estándares de la calidad de la salud para toda la población en línea con los intereses políticos y poder causar un impacto plausible en la satisfacción de ambas partes; estos estándares deben contemplar los factores de necesidad como un elemento base.

Los resultados de la encuesta nacional de ocupación y empleo, con cifras del segundo trimestre de 2019, el INEGI menciona que un total de 20.6 millones de estos trabajadores, los cuales constituyen el $55.1 \%$ del total, tienen acceso a instituciones de salud como prestación por su trabajo (INEGI, 2019) a pesar de que esta cifra ha aumentado, todavía existe un $44.9 \%$ de trabajadores que no tienen esta prestación, por lo que deben acudir a servicios privados a instituciones de la Secretaria de Salud.

Esta situación aumenta la presión sobre los servicios médicos de la SSA, que también tienen diferentes áreas de oportunidad, entre ellas la cobertura del servicio a toda la población.

Algunas de las ventajas de la obtención de las Áreas con mayor aptitud que se muestran en este resultado, son que se han tomado en consideración las necesidades de la población, que nunca se toman en consideración, a pesar de que serán ellos los que se beneficien con la implementación, además de tomar en consideración criterios físicos, que pueden influir en menores costos económico, debido a la mejor pendiente y a la cercanía con las vías que comunican los diferentes poblados.

\section{Conclusión}

Los resultados obtenidos en la presente investigación permiten identificar zonas de oportunidad para la localización de nuevos servicios médicos de carácter público, aportando soluciones a un problema real, tangible y de gran interés considerando que históricamente la localización de este tipo de servicios en México se ha ajustado a criterios que distan de las necesidades de la población, siendo los grupos más desfavorecidos los que padecen la falta de acceso a este tipo de servicios.

Los municipios como Almoloya de Juárez, Calimaya, Lerma, Otzolotepec y Zinacantepec son los que registran áreas con mayor aptitud para los nuevos servicios médicos estos mismos municipios son los que registran una mayor falta de cobertura, sobre todo en las áreas periféricas o conurbadas, que a pesar de ser parte de un zona metropolitana hay localidades eminentemente rurales y algunas además tienen población dispersa que no cubren con el número mínimo para asignar una nueva unidad médica, por lo que el acceso a los servicios no les llega de manera adecuada, salvo algunas ocasiones en donde se envían unidades móviles.

Lo antes mencionado da pauta a recomendar la revisión de la normatividad vigente en aras de que toda la población cuente con un servicio médico que promueva el funcionamiento igual para todos, del mismo modo implementar estrategias focalizadas para mejorar la calidad de salud de las personas. 
Santana Castañeda, G., Aguilar Martínez, A.G. (2020). Sitios candidatos para nuevos servicios médicos utilizando técnicas de evaluación multicriterio, en la Zona Metropolitana de Toluca, México. GeoFocus, Revista Internacional de Ciencia y Tecnología de la Información Geográfica, 26, 139-162. http://dx.doi.org/10.21138/GF.602

Con relación a la metodología, el análisis de las diferentes técnicas antes mencionadas fue de gran ayuda, así como la utilización combinada de ellas y el proceso para obtener los resultados considera una solución integral incluyendo un apartado sobre las necesidades de la población, apoyando la población que se encuentra en mayor desventaja por sus situaciones multi carenciales.

La información generada, podrá permitir a los que toman decisiones profundizar en su conocimiento de la realidad del territorio, y emprender acciones teniendo en cuenta las necesidades e intereses de la población.

\section{Referencias bibliográficas}

Abdullah, L. (2013). Fuzzy multi criteria decision making and its applications: A brief review of category. Procedia - Social and Behavioral Sciences, 131-136.

Abdullah, L., \& Jamal, J. (2011). Determination of Health-Related Quality of Life Indicators: A Fuzzy Decision-Making Method. Applied Research in Quality of Life, 349-361.

Barredo Cano, J., \& Bosque Sendra, J. (1999). Multicriteria evaluation methods for ordinal data in a GIS environment. España: RAMA.

CHURCH, Richard L. Location modelling and GIS. Geographical information systems, 1999, vol. 1, p. 293-303.

Colson, G., \& De Bruin, C. (1989). Models and methods in multiple criteria decisions making. Pergamon Press.

Diamond, J., \& Wright, J. (1988). Design of an Integrated Spatial Information System for Multiobjective Land-Use Planning. Environment and Planning. Environment and Planning B Planning and Design 15(2):205-214 · April 1988

Eastman, J., Jiang, H., \& Toledano, J. (1998). Multi-Criteria and Multi-Objective Decision Making for Land Allocation Using GIS. In Multi-Criteria Analysis for Land-Use Management, Environment and Management (pp. 227-251). E. Beinat and P. Nijkamp.

Galacho J., F., \& Ocaña O., C. (2006). Tratamiento con SIG y técnicas de evaluación multicriterio de la capacidad de acogida del territorio para usos urbanisticos: residenciales y comerciales. Departamento de Geografía, Universidad de Málaga, 1509-1525.

Gómez, M., \& Barredo, C. (2005). Sistemas de información geográfica y evaluación multicriterio en la ordenación del territorio. Madrid: RA-MA.

Hwang, C., \& Yoon, K. (1981). Multiple Attribute Decision Methods and Applications. Springer: Berlin Heidelberg.

INEGI (2019) "Resultados de la encuesta nacional de ocupación y empleo. Cifras durante el segundo trimestre de 2019" Comunicado de prensa número 399/19. 14/08/2019. Disponible en: https://www.inegi.org.mx/contenidos/saladeprensa/boletines/2019/enoe_ie/enoe_ie2019_08.pdf 


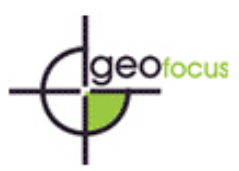

Santana Castañeda, G., Aguilar Martínez, A.G. (2020). Sitios candidatos para nuevos servicios médicos utilizando técnicas de evaluación multicriterio, en la Zona Metropolitana de Toluca, México. GeoFocus, Revista Internacional de Ciencia y Tecnología de la Información Geográfica, 26, 139-162. http://dx.doi.org/10.21138/GF.602

Jankowski, P. (1995). Integrating geographical information systems and multiple criteria decisionmaking methods. International Journal of Geographical Information Systems, 251-273.

Janssen, R., \& Rietveld, P. (1990). Multi-criteria analysis and geographical information systems: an application to agricultural landuse in the Netherlands. Amsterdan: Scholten H. J., Stillwell J. C. H.

Malczewski, J. (1999). Gis an multicriteria decisión analysis. Nueva York: J. Wiley.

Nijkamp, P., \& Van Delf, A. (1977). Multicriteria analysis and regional planning. The Netherlands: Martinus Nijhoff.

OMS (2008) "La atención primaria de salud, más necesaria que nunca" Disponible en línea: https://www.who.int/whr/2008/summary/es/

Pomerol, J., \& Barba R., S. (1993). Choix multicritère dans l'entreprise: principe et pratique. Paris: Hermes.

Saaty, Thomas L. How to make a decision: the analytic hierarchy process. European journal of operational research, 1990, vol. 48, no 1, p. 9-26.

Saaty T., 2014 El proceso analítico jerárquico. La toma de decisiones en un mundo complejo RWS publications Pittsburgh.

Secretaria de salud. (1995). Modelo de atención a la salud para población abierta. México.

Secretaria de salud. (2006). Modelo integrador de atención a la salud (MIDAS). México.

SEDESOL. (1999). Tomo II: Salud y asistencia social. En sistema normativo de equipamiento urbano, Secretaria de Salud. México: SEDESOL.

Smith, D. (1977). Geografía humana. Barcelona: Oikos-Tau.

Tseng, T., \& Cerry, M. (1992). A new algorithm for fuzzy multicriteria decision making. Department of industrial engineering, 45-66.

Zeleny, M., \& Cochrane, J. L. (1973). Multiple criteria decisions making. University of South Carolina Press. 